\title{
Integrated Computational Materials Engineering of Gamma Titanium Aluminides for Aerospace Applications
}

\author{
Matthew S. Dahar ${ }^{1}$, Sesh A. Tamirisakandala ${ }^{1}$, John J. Lewandowski ${ }^{2}$ \\ ${ }^{1}$ ARCONIC, 1000 Warren Ave, Niles OH 44446 USA \\ ${ }^{2}$ Department of Materials Science \& Engineering, Case Western Reserve University, Cleveland OH 44106 USA
}

Sesh.Tamirisakandala@Arconic.com

\begin{abstract}
$\underline{\text { Abstract }}$
Although the benefits of titanium aluminides for intermediate service temperature applications were well conceived and significant research and development activities were conducted in the past four decades, they remained as developmental materials due to barriers associated with melting, processing, scale-up, and cost. Demanding requirements of efficient aero-engines and extensive risk reduction demonstrations paved the path for commercial introduction of gamma titanium aluminides. The single most attractive current application is for low pressure turbine blades (LPTBs) in advanced aero-engines replacing conventionally cast nickel superalloys. This paper provides an overview of recent progress, producibility challenges, and opportunities. The successful journey of gamma $(\gamma)$ TiAl LPTB development from laboratory demonstrations to production insertions in mass-produced commercial jet engines will be described. Collaboration and integrated product development were identified as the most critical needs for rapid maturation and implementation of $\gamma$-TiAl into aerospace applications. An integrated computational materials engineering modeling framework and toolsets developed under a collaborative US Air Force Metals Affordability Initiative project between industry, government, and academia will be illustrated. Model-based optimization of material and processing for achieving desired performance goals will be highlighted.
\end{abstract}

\section{Introduction}

Intermetallic compounds formed between light elements Tic and $\mathrm{Al}$ are attractive because of their low density and good elevated temperature strength. However, the formation of intermetallic compounds reduces the symmetry of the parent metal lattice, restricting available deformation modes. These restrictions are usually manifested as increased strength (at room and moderate temperatures), reduced ductility and fracture toughness. Historically, the risks associated with reduced ductility and fracture toughness have been viewed as outweighing the benefits of increased strength. Consequently, the use of intermetallic compounds in structural applications has been very limited. A class of $\gamma$-TiAl compositions based on $42-48$ at $\% \mathrm{Al}$, plus additional elements as identified in Figure 1, evolved over the past 40 years that offer attractive specific strengths compared to high-density nickel superalloys/steels up to $750^{\circ} \mathrm{C}$ service temperatures (Figure 1). These compositions can be broadly classified into two groups alloys that contain greater than 85 vol. $\% \gamma$ phase (peritectic-solidifying) and alloys that contain less than 75 vol $\% \gamma(\beta$ solidifying). These $\gamma$-TiAl materials have found a niche application in the form of low pressure turbine blades (LPTBs) in next generation jet engines that are providing higher efficiencies, leading to reduced fuel consumption and reduced emissions $[1,2]$.

The product introduction of any new material and/or process for a critical aerospace application is a big undertaking. It requires the successful transition from lab/pilot scale activities to those routinely conducted in a production setting. Insertion of the first $\gamma$-TiAl aerospace application, Ti-48Al-2Nb-2Cr (Ti-48-2-2) LPTB on the General Electric GEnx engine, took over three decades from alloy development, component trials, engine testing, material database generation, qualification, specifications generation, and finally to serial production (Figure 2). Contributing reasons for a such a long time from development to implementation include the intrinsic brittle nature of $\gamma$-TiAl, manufacturing difficulties, high cost, and economic hardships experienced in production. Large scatter in mechanical properties has been a significant problem that demands tight control of chemical composition over narrow ranges. Microstructure and texture heterogeneities emanating from solidification, phase transformations, and thermal processing also pose concerns. Investment cast LPTBs of Ti-48-2-2 have been successfully flying on over 1,000 GE GEnx engines in service on commercial aircrafts (Boeing 787 and 747) since 2010. The lessons learned from the first application and production experiences of Ti-48-2-2 were implemented in the introduction of a second application in CFM LEAP engines that are powering the next generation of fuel-efficient single-aisle commercial aircrafts (Boeing 737 MAX, Airbus A320neo, and COMAC C919). Unprecedented demand for LEAP engines ( 15,000 engines on order) and rapid delivery times required the development of innovative manufacturing methods. As illustrated in Figure 3, collaborating with SAFRAN, direct machining of LPTBs from cast slugs was developed by Arconic to enable affordable production and on-time delivery. 

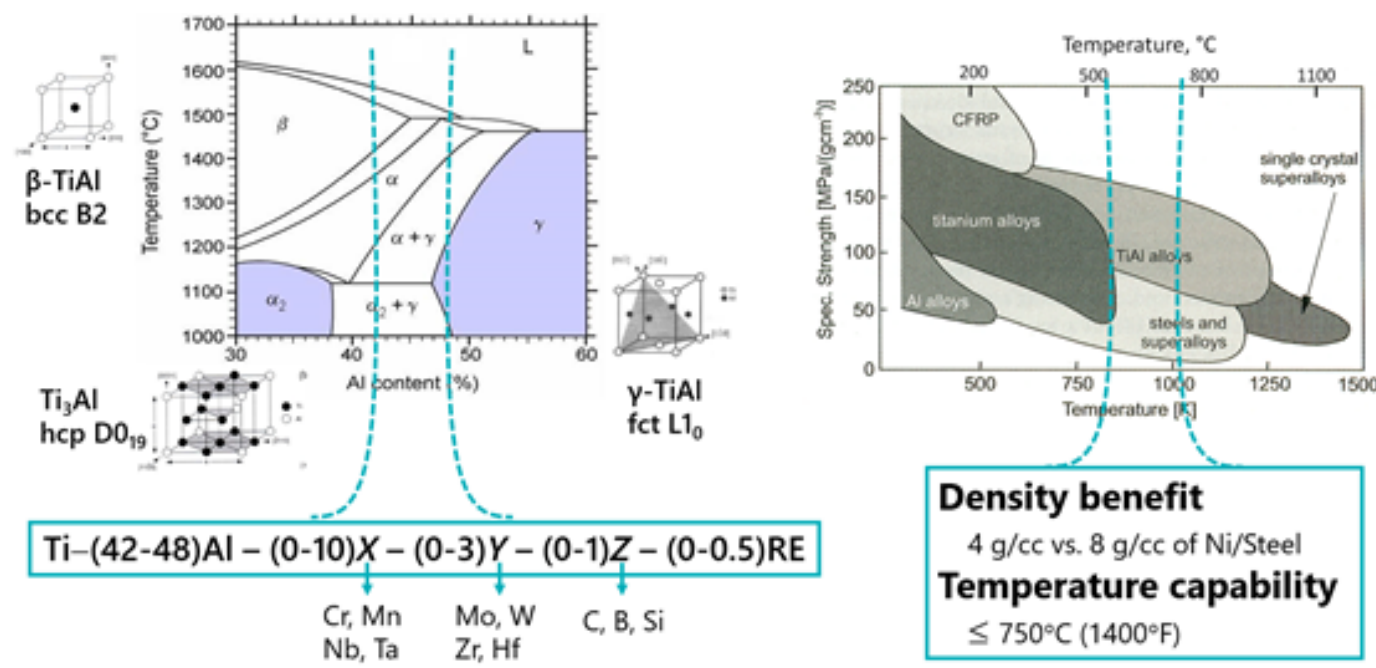

Figure 1: Gamma titanium aluminide composition design space (left) and specific strength comparison with other structural metals.

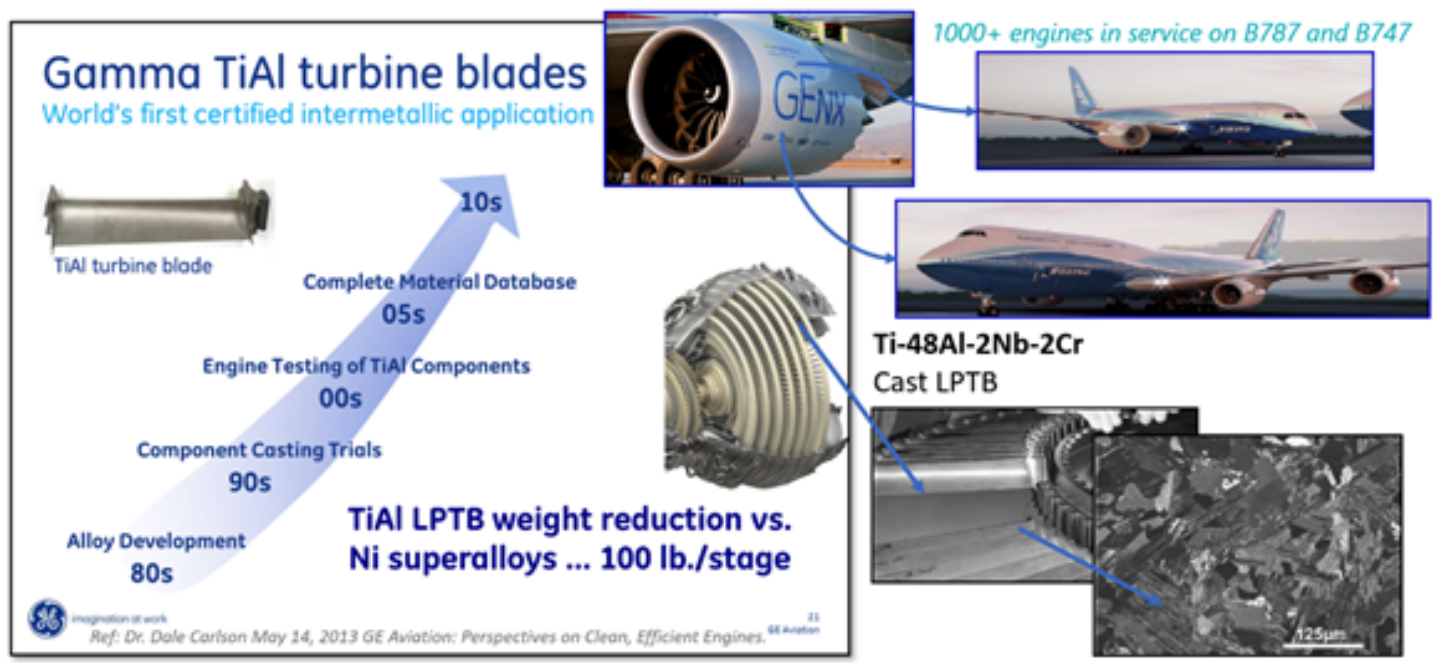

Figure 2: Gamma titanium aluminide low pressure turbine blades first commercial application from concept to flight. 

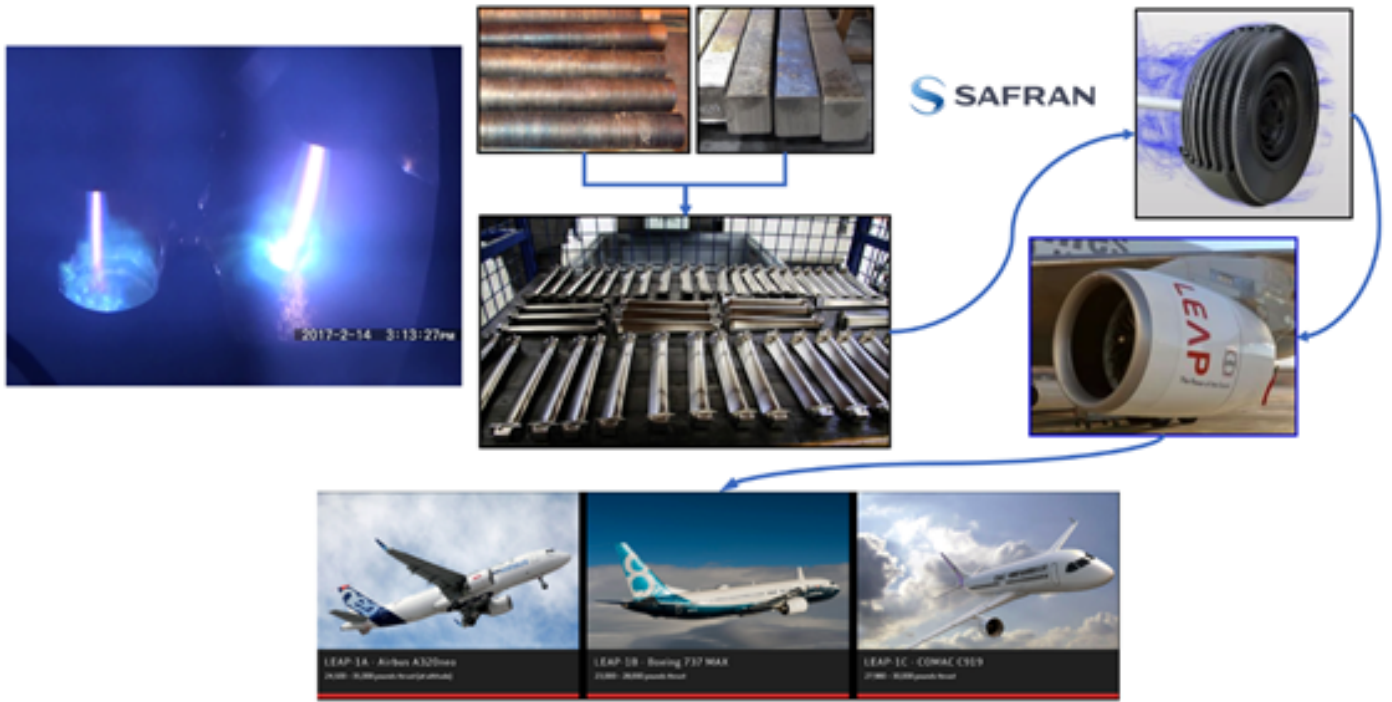

Figure 3: Transition of cast LPT $\gamma$-TiAl blades from GEnx engine to large volume CFM LEAP engine.

Pratt \& Whitney recently introduced a geared turbo fan (GTF) engine in which the fan shaft is connected to the LPT through a gear box that reduces the fan rotational speed relative to the LPT. This permits the LPT to operate at more optimum (higher) speeds, which has associated efficiency gains. To meet the property requirements of higher rotational speeds of GTF LPT, Ti-43.5Al-4Nb$1 \mathrm{Mo}-0.1 \mathrm{~B}$ (TNM), was developed in the 2000s (Figure 4). This $\beta$-solidifying $\gamma$-TiAl exhibits a refined as-cast grain size due to trace $\mathrm{B}$ additions and a higher volume fraction of high-temperature $\beta$-TiAl (disordered body centered cubic structure) phase. This microstructure evolves due to the addition of balanced amounts of $\mathrm{Nb}$ and Mo which imparts enhanced hot workability, thereby enabling the introduction of forged TNM blades. The ability to minimize the volume fraction of $\beta_{\mathrm{O}}$-TiAl (ordered B2 structure) phase via a post-forging heat treatment provides balanced properties for TNM at room and service temperatures [3].
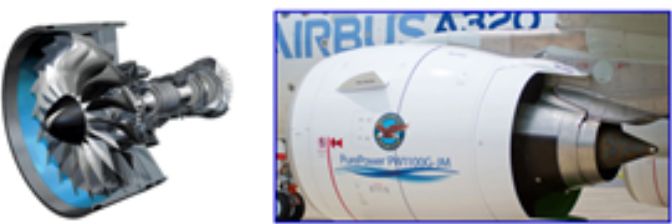

High rotational speeds of LPTB in GTF

$\rightarrow$ More pronounced benefits of weight reduction

$\rightarrow$ Ti-43Al-4Nb-1Mo-0.1B Forged LPTB for higher $\sigma$
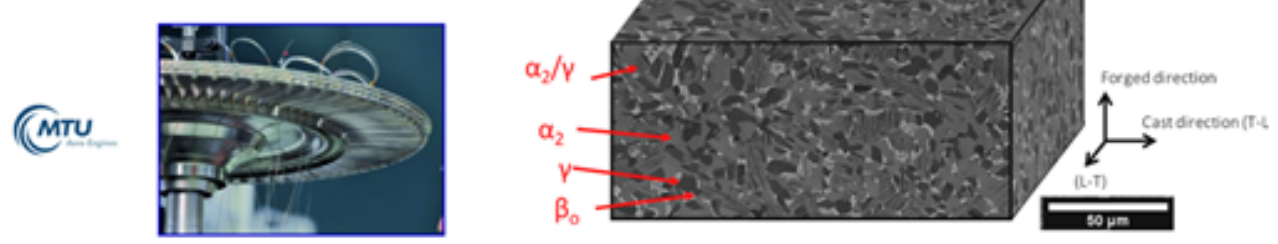

Ability to balance static and dynamic property combinations

Figure 4: Key attributes of wrought $\gamma$-TiAI TNM currently in use for PW GTF engine LPT blades.

\section{ICME Model-based Enhanced Development}

With an objective of accelerated technology and manufacturing readiness levels maturation of $\gamma$-TiAl, an activity integrated project team (AIPT) was formed under the auspices of the US Air Force Metals Affordability Initiative (MAI) consisting of original equipment manufacturers OEMs (Boeing, GE Aviation, Honeywell Aerospace, Pratt \& Whitney, and Rolls Royce), material and component producers (Arconic, ATI, and PCC), modelers (CompuTherm LLC, DREAM3D), and universities (Case Western 
Reserve, Georgia Institute of Technology, and Iowa State). The goal of this AIPT project was to develop integrated computational materials engineering (ICME) models and tools that enable rapid design and prediction capabilities. The program utilized an ICME methodology that incorporated thermodynamic and phase distribution effects through database development, novel image processing techniques for digital representation of microstructures, microstructure-property correlations using artificial neural networks, meso-scale loading effects through efficient crystal plasticity models, macro-scale simulation of component fabrication using constitutive datasets, and micro-mechanistic fatigue and fracture behavior understanding. These developments enhanced both the primary metal production and subsequent component manufacturing. High-fidelity commercially available modeling tools were utilized for development of components via casting, isothermal/hot-die forging, and complex heat treatment processes. The program utilized sub-scale and full-scale demonstrations to develop, test, and evaluate cast and wrought $\gamma$-TiAl components. The results were used to pinpoint capabilities to assist OEMs in identifying opportunities for use on future systems. A few examples of the models and tools developed under this project are highlighted below.

\subsection{Thermodynamic Model}

A CALPHAD thermodynamic model for $\gamma$-TiAl was developed by CompuTherm LLC to predict multi-component pseudo-binary and more complex phase diagrams comprising 16 elements (Al, B, C, Cr, Mn, Mo, N, Nb, O, Sn, Si, Ta, Ti, V, W, and Zr) using a Pandat database [4]. Key binary and ternary systems were developed by comparing with experimental data of $\gamma$-solvus temperature and phase fractions. Preliminary work was also conducted on the development of a molar volume database for Ti-Al-Mo and Ti$\mathrm{Al}-\mathrm{Nb}$ ternary systems for density calculations, which were combined with thermodynamic calculations for the selection of desired phases in alloy design.

\subsection{Solidification Model}

GE developed a 3D solidification model for $\gamma$-TiAl by adapting codes previously developed for fcc structures to bcc systems. This physics-based solidification model was coupled with commercially available tools such as Pandat for thermodynamic calculations and casting simulation tools such as ProCast for solidification conditions. The solidification model can predict microstructure (nucleation, grain size, grain orientation, primary and secondary dendrite arm spacings) and microsegregation (element partitioning and diffusion) in $\gamma$-TiAl castings. These predictions are valuable in understanding how the selection of average alloy compositions can produce potentially different solidification paths, in addition to helping to define future alloy composition specification boundaries.

\subsection{Microstructure-Property Correlation Model}

Microstructural features of multi-phase $\gamma$-TiAl span several orders of magnitude length scales and exhibit different morphologies. Image processing, segmentation, and quantification of $\gamma$-TiAl microstructures using conventional methods is non-trivial. A team from Iowa State University developed custom recipes for segmentation and quantification of various phase features including spatial variations of $\gamma$-TiAl microstructures in a commercial image processing software (Material Image Processing and Automated Reconstruction MIPAR [5]). A neural network model was developed linking microstructural features and mechanical properties.

\subsection{Crystal Plasticity Model}

Georgia Institute of Technology developed a crystal plasticity model incorporating microstructure and texture statistical volume elements (SVEs) of $\alpha_{2}-\mathrm{Ti}_{3} \mathrm{Al}, \gamma$-TiAl, $\beta$-TiAl and $\alpha_{2}+\gamma$ lamellar colony microconstituents [6]. The ICME modeling workflow used a Python pipeline that couples DREAM.3D synthetic microstructure representation with a user defined Abaqus material file (UMAT). Representative experimental microstructure and texture measurements from forgings were input to construct multiple SVEs for finite element modeling and successful prediction of bulk mechanical properties.

\subsection{Micro-mechanistic Fatigue and Fracture Model}

The Case Western Reserve University and Arconic team generated fatigue and facture data on as-cast, cast + HIP, and forged $\gamma$ TiAl material in different orientations, as shown in Figure 5. Key parameters such as the fatigue thresholds, overload toughnesses, Paris regime exponents, and plastic zone sizes were databased to develop a micro-mechanistic understanding [7]. A data-driven model was then developed by linking microstructure features/scale to crack tip plastic zone size and crack growth rate. 

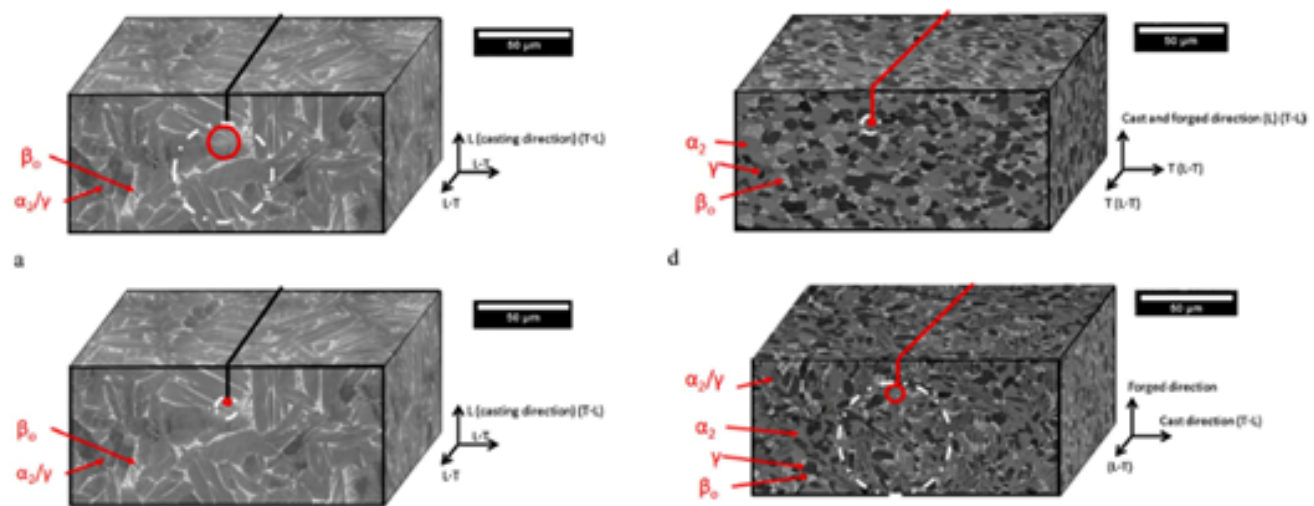

d
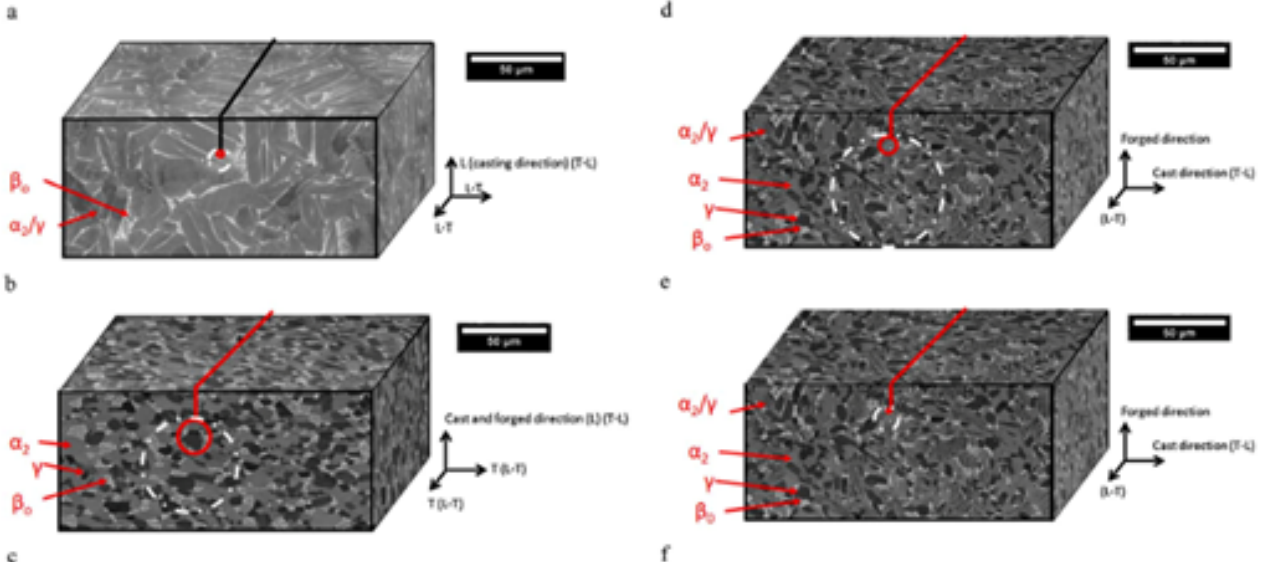

Figure 5: Maximum monotonic (left) and cyclic (right) plastic zone sizes calculated for fatigue crack growth experiments conducted at $R$ $=0.1$ on TNM in various microstructure conditions noted. The solid line represents the calculated plastic zone size at the fatigue threshold and the dashed line for catastrophic overload.

\section{Future Opportunities}

Several future opportunities for $\gamma$-TiAl listed in Table 1 exist with the business case primarily driven by significant weight reduction potential. However, each of these opportunities come with challenges and risks that could be overcome by the utilization of ICME tools and collaborative development. High pressure compressor blades have the same benefits as LPTBs, but must overcome the brittle nature of the material and ability to produce thinner cross-section parts to tight tolerances. Exhaust nozzles are another promising opportunity, but technology maturation to scale-up and produce large-size parts is required. Space systems are also a potential opportunity that require better fabrication methods. Automotive and additive manufacturing present significant opportunities for $\gamma$-TiAl, but affordability is a key barrier for entry. Increasing the service temperature capability of $\gamma$-TiAl via improved alloy designs and application of surface coatings is an active area of research [8].

\section{Conclusions}

The first commercial $\gamma$-TiAl aerospace application took 30 years from concept to implementation, overcoming significant challenges. Recognizing the critical need for rapid development and insertion, an activity integrated project team comprising engine manufactures, material and component producers, small businesses, government labs, and universities was formed. A community-wide collaborative effort was conducted to develop integrated computational materials engineering (ICME) models and tools that support accelerated material and process maturation for future applications of $\gamma$-TiAl.

Table 1: Potential applications, benefits, and challenges for gamma TiAl. 


\begin{tabular}{|c|c|c|}
\hline Application & Benefits & Challenges \\
\hline High Pressure Compressor Blades & Same benefits as LPTB & $\begin{array}{l}\text { Impact damage tolerance } \\
\text { Thinner crosss-sections than LPTB } \\
\text { Cost }\end{array}$ \\
\hline Exhaust nozzles (static) & Weight reduction & Large size \\
\hline Structural components (static) & $\begin{array}{l}\text { Near-net, large, } \\
\text { irregular, unsymmetrical } \\
\text { shape castings }\end{array}$ & Weld repair \\
\hline Space systems & $\begin{array}{l}\text { Light weight } \\
\text { High section modulus }\end{array}$ & Fabrication \\
\hline Automotive exhaust valves & $\begin{array}{l}\text { Weight reduction } \\
\text { Fuel economy }\end{array}$ & Cost \\
\hline Additive Manufacturing & Design freedom & $\begin{array}{l}\text { Reliability } \\
\text { Cost }\end{array}$ \\
\hline
\end{tabular}

\section{$\underline{\text { Acknowledgements }}$}

The support from US Air Force Metals Affordability Initiative (Agreement Order No. FA8650-15-2-5201 AO\#43) for ICME models and tools development is greatly appreciated.

\section{$\underline{\text { References }}$}

1. G. Lutjering and J.C. Williams, Titanium, 2nd ed., Springer-Verlag, New York (2007) 354-366.

2. Aerospace Materials and Applications, B.N. Bhat, ed., https://arc.aiaa.org/doi/book/10.2514/4.104893, American Institute of Aeronautics and Astronautics, Inc. Reston VA (2018) 602-603.

3. H. Clemens W. Smarsly V. Güther, S. Mayer, Advanced Intermetallic Titanium Aluminides, Proceedings of the 13th World Conference on Titanium, TMS (2015) 1189-1200.

4. F. Zhang et al., "Accelerated Design of Gamma-TiAl Alloys by High Throughput Calculation”, MRS Fall Meeting, November 2018.

5. http://www.mipar.us

6. A. Muth et al., "Microstructure-Sensitive Computational Scheme for Fatigue Resistance of Gamma-TiAl TNM Alloys", Materials Science and Technology 2017, TMS, Salt Lake City, UT.

7. M. S. Dahar, S. A. Tamirisakandala, and J. J. Lewandowski, "Evolution of fatigue crack growth and fracture behavior in gamma titanium aluminide Ti-43.5Al-4Nb-1 Mo-0.1B (TNM) forgings,” Int. J. Fatigue, vol. 111 (2018) 54-69.

8. YW Kim SL Kim, “Advances in Gammalloy Materials-Processes-Application Technology: Successes, Dilemmas, and Future", JOM (2018) 70: 553 